\title{
PLANNING IDENTIFICATION EXPERIMENTS FOR CELL SIGNALING PATHWAYS: AN NF $\kappa$ B CASE STUDY
}

\author{
KRZYSZTOF FUJAREWICZ \\ Institute of Automatic Control \\ Silesian University of Technology, Akademicka 16, 44-100 Gliwice, Poland \\ e-mail: krzysztof.fujarewicz@polsl.pl
}

\begin{abstract}
Mathematical modeling of cell signaling pathways has become a very important and challenging problem in recent years. The importance comes from possible applications of obtained models. It may help us to understand phenomena appearing in single cells and cell populations on a molecular level. Furthermore, it may help us with the discovery of new drug therapies. Mathematical models of cell signaling pathways take different forms. The most popular way of mathematical modeling is to use a set of nonlinear ordinary differential equations (ODEs). It is very difficult to obtain a proper model. There are many hypotheses about the structure of the model (sets of variables and phenomena) that should be verified. The next step, fitting the parameters of the model, is also very complicated because of the nature of measurements. The blotting technique usually gives only semi-quantitative observations, which are very noisy and collected only at a limited number of time moments. The accuracy of parameter estimation may be significantly improved by a proper experiment design. Recently, we have proposed a gradient-based algorithm for the optimization of a sampling schedule. In this paper we use the algorithm in order to optimize a sampling schedule for the identification of the mathematical model of the NF $\kappa \mathrm{B}$ regulatory module, known from the literature. We propose a two-stage optimization approach: a gradient-based procedure to find all stationary points and then pair-wise replacement for finding optimal numbers of replicates of measurements. Convergence properties of the presented algorithm are examined.
\end{abstract}

Keywords: cell signaling pathways, experiment design, parameter estimation.

\section{Introduction}

One of possible approaches to the modeling of cell signaling pathways is to use a set of nonlinear ODEs (de Jong, 2002). In order to estimate unknown parameters of such a model, several experiments are performed, during which concentrations of part of variables are measured at rare discrete time moments. Usually, different blotting techniques or DNA microarrays are used. Proper experiment design plays a fundamental role in model identification and validation (Box and Lucas, 1959). In this work, we focus on choosing optimal time moments for measurements (sampling). This problem has been investigated in the related literature. Usually, to solve the problem, a matrix of correlation coefficients between sensitivities of measurements with respect to identified parameters is calculated (Jacquez and Greif, 1985; Jacquez, 1998). Then one tries to choose such time moments for which the sensitivities are "less correlated". This is done by determining the so-called Fisher information matrix (FIM) (D’Argenio, 1981; DiStefano, 1981). A similar approach is also used for identifiability checking (Jacquez and Greif, 1985; Jacquez, 1998). Based on the Cramer-Rao inequality, one can show that under some assumptions the inverse of the FIM is a lower bound to the covariance matrix of parameter estimates. There are several possible approaches to the minimization of such a covariance matrix. The most widely used technique is to maximize the determinant of the FIM. This is the so-called D-optimal experiment design (Fedorov, 1972; D’Argenio, 1981; DiStefano, 1981; Goodwin and Payne, 1977).

To speed up the procedure, the gradient of the FIM determinant can be calculated. This was done, for example, by Tod and Rocchisani (1997) under the assumption that the model is given as a function of time. Unfortunately, in the case of cell signaling/metabolic pathways, mathematical models take the form of sets of nonlinear ODEs and there is no analytical form of time-domain solutions. Recently (Fujarewicz, 2007; 2008), we derived formulas for the calculation of the gradient of the FIM in the space of sampling times for the model given by ODEs 
in state space.

D-optimization requires prior assumptions about nominal parameter values. This is an unrealistic assumption (simply because we perform the experiment in order to estimate these parameters). Of course it is possible to conduct a set of experiments which, one by one, improve the parameter estimates. Such an approach is called sequential design (Chernoff, 1972; D’Argenio, 1981; DiStefano, 1981). Another (more realistic) approach is to assume some distributions for nominal parameters, not constant values. In this case, when the gradient is obtainable, the stochastic gradient approach can be applied. This is the so-called ED-optimization (Pronzato and Walter, 1985).

In this work we use formulas for the gradient derived by Fujarewicz $(2007 ; 2008)$, in order to perform D-optimization of an experiment plan for a mathematical model of the $\mathrm{NF} \kappa \mathrm{B}$ regulatory module proposed by Lipniacki et al. (2004). We show that gradient-based Doptimization does not guarantee obtaining a globally optimal design. Nevertheless, using this procedure it is possible to find properly all of the so-called stationary points. This property is then used in formulating a two-stage optimization procedure. The next stage, after finding all stationary points, is to find their multiplicities which are the numbers of replicates of measurements performed at the same time (stationary point). The convergence properties of the two stages are examined and finally the best found D-optimized design for the $\mathrm{NF} \kappa \mathrm{B}$ signaling pathway is presented.

\section{Mathematical model of the NF $\kappa B$ regulatory module}

In this section we briefly present a mathematical model of $\mathrm{NF} \kappa \mathrm{B}$ regulatory module proposed by Lipniacki et al. (2004). The NF $\kappa \mathrm{B}$ transcription factor regulates numerous genes that play important roles in inter- and extracellular signaling. It governs many cell processes such as cellular stress responses, cell growth, survival and apoptosis.

The model takes the form of 15 first order nonlinear differential equations:

$$
\begin{aligned}
\dot{x}_{1}= & k_{\text {prod }}-k_{d e g} x_{1}-k_{1} u x_{1}, \\
\dot{x}_{2}= & k_{1} u x_{1}-k_{3} x_{2}-k_{2} u x_{2} x_{8}-k_{d e g} x_{2}-a_{2} x_{2} x_{10} \\
& +t_{1} x_{4}-a_{3} x_{2} x_{13}+t_{2} x_{5}, \\
\dot{x}_{3}= & k_{3} x_{3}+k_{2} u x_{2} x_{8}, \\
\dot{x}_{4}= & a_{2} x_{2} x_{10}-t_{1} x_{4}, \\
\dot{x}_{5}= & a_{3} x_{2} x_{13}-t_{2} x_{5} \\
\dot{x}_{6}= & c_{6 a} x_{13}-a_{1} x_{6} x_{10}+t_{2} x_{5}-i_{1} x_{6}, \\
\dot{x}_{7}= & i_{1} k_{v} x_{6}-a_{1} x_{7} x_{11}, \\
\dot{x}_{8}= & c_{4} x_{9}-c_{5} x_{8},
\end{aligned}
$$

$$
\begin{aligned}
\dot{x}_{9}= & c_{2}+c_{1} x_{7}-c_{3} x_{9}, \\
\dot{x}_{10}= & -a_{2} x_{2} x_{10}-a_{1} x_{6} x_{10}+c_{4 a} x_{12}-c_{5 a} x_{10} \\
& -i_{1 a} x_{10}+e_{1 a} x_{11}, \\
\dot{x}_{11}= & -a_{1} x_{7} x_{11}+i_{1 a} k_{v} x_{10}-e_{1 a} k_{v} x_{11}, \\
\dot{x}_{12}= & c_{2 a}+c_{1 a} x_{7}-c_{3 a} x_{12}, \\
\dot{x}_{13}= & a_{1} x_{6} x_{10}-c_{6 a} x_{13}-a_{3} x_{2} x_{13}+e_{2 a} x_{14}, \\
\dot{x}_{14}= & a_{1} x_{7} x_{11}-e_{2 a} k_{v} x_{14}, \\
\dot{x}_{15}= & c_{2 c}+c_{1 c} x_{7}-c_{3 c} x_{15} .
\end{aligned}
$$

In the model (1), state variables are concentrations of proteins, complexes of proteins or their transcripts as follows:

$x_{1}$ : IKK kinase in the neutral state (IKKn),

$x_{2}:$ IKK in the active state (IKKa),

$x_{3}$ : IKK in the inactive state (IKKi),

$x_{4}:(\mathrm{IKKa}-\mathrm{I} \kappa \mathrm{Ba})$ complexes,

$x_{5}:(\mathrm{IKKa}-\mathrm{I} \kappa \mathrm{Ba}-\mathrm{NF} \kappa \mathrm{B})$ complexes,

$x_{6}$ : free cytoplasmic $\mathrm{NF} \kappa \mathrm{B}$,

$x_{7}$ : free nuclear $\mathrm{NF} \kappa \mathrm{B}$,

$x_{8}:$ A20 protein,

$x_{9}:$ A20 transcript,

$x_{10}$ : free cytoplasmic $\mathrm{I} \kappa \mathrm{B}$ a protein,

$x_{11}$ : free nuclear $\mathrm{I} \kappa \mathrm{B}$ a protein,

$x_{12}: \mathrm{I} \kappa \mathrm{Ba}$ transcript,

$x_{13}$ : cytoplasmic $(\mathrm{I} \kappa \mathrm{Ba}-\mathrm{NF} \kappa \mathrm{B})$ complexes,

$x_{14}$ : nuclear $(\mathrm{I} \kappa \mathrm{Ba}-\mathrm{NF} \kappa \mathrm{B})$ complexes,

$x_{15}$ : control gene transcript,

$u$ : TNF stimulation (0 or 1$)$.

Parameters of the model (1) were fitted roughly (manually) in the work of Lipniacki et al. (2004), based on measurements taken from previous articles (Hoffman et al., 2002; Lee et al., 2000) showing blot experiments images. In the work of Fujarewicz et al. (2007), parameters of the same model were fitted once more using the so-called generalized back propagation through time (GBPTT) algorithm (Fujarewicz and Galuszka, 2004), giving more accurate parameter estimates. Results of simulation for parameters obtained by Fujarewicz et al. (2007) are presented in Fig. 1 However, the accuracy of parameter estimates depends on the chosen estimation procedure. But the main source of variation in the obtained estimates depends on the conducted wet-lab experiment. In the research by Hoffman et al. (2002) and Lee et al. (2000), only part of 15 variables was measured: $x_{2}$, 

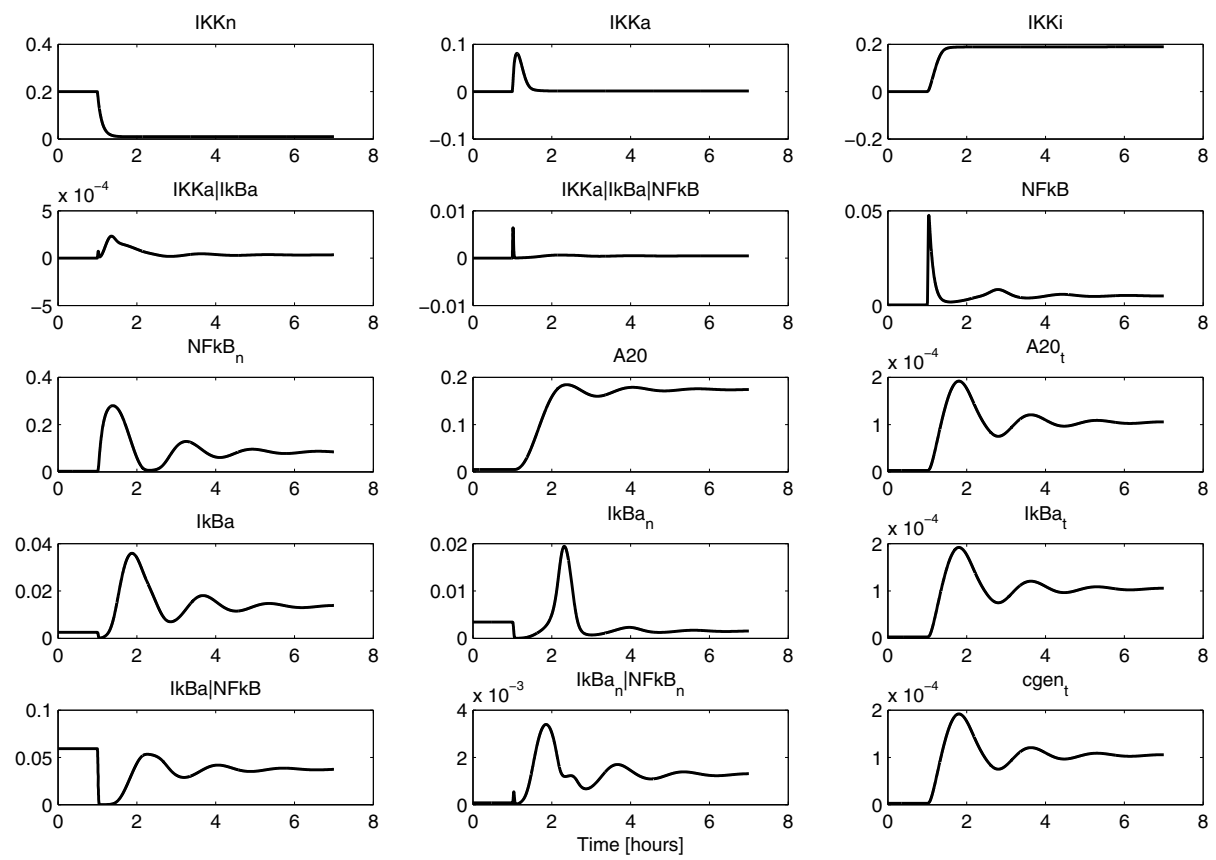

Fig. 1. Results of simulation of the NF $\kappa \mathrm{B}$ mathematical model.

$x_{7}, x_{9}, x_{12}$ and total cytoplasmic $\mathrm{I} \kappa \mathrm{Ba}: x_{10}+x_{13}$. This gives a linear output equation

$$
y=C x,
$$

where $C$ is the matrix with almost all terms equal to zero except $(i, j)=(1,2),(2,7),(3,9),(4,12),(5,10)$ and $(5,13)$.

\section{Problem formulation}

Let us consider a model of a cell signaling pathway described by a set of non-linear ODEs:

$$
\dot{x}=f(x, u, \theta), \quad x(0)=x_{0},
$$

where $x$ is a vector of state variables, $u$ is an input signal and $\theta \in \mathbb{R}^{p}$ is a vector of identified parameters. The output equation is as follows:

$$
y=g(x)
$$

For simplicity of notation, let us assume there is only one output variable which is measured at times $t_{1}, t_{2}, \ldots, t_{n}$ giving instantaneous values:

$$
y\left(t_{i}\right)=g\left(x\left(t_{i}\right)\right)=g_{i}, \quad i=1,2, \ldots, n .
$$

After performing experiments, one obtains

$$
z\left(t_{i}\right)=y\left(t_{i}\right)+\varepsilon_{i}, \quad i=1,2, \ldots, n,
$$

where $\varepsilon_{i}$ is an error of zero mean and variance $\sigma_{i}^{2}$. We assume that we have initial (rough) estimation of parameters $\theta_{1}^{0}, \theta_{2}^{0}, \ldots, \theta_{n}^{0}$ for which the measured variable (5) takes values $g_{1}^{0}, g_{2}^{0}, \ldots, g_{n}^{0}$. We build the sensitivity matrix as follows:

$$
G=\left[\begin{array}{ccc}
\frac{\partial g_{1}^{0}}{\partial \theta_{1}} & \cdots & \frac{\partial g_{1}^{0}}{\partial \theta_{p}} \\
\vdots & \ddots & \vdots \\
\frac{\partial g_{n}^{0}}{\partial \theta_{1}} & \cdots & \frac{\partial g_{n}^{0}}{\partial \theta_{p}}
\end{array}\right]
$$

and the Fisher information matrix

$$
I=G^{T} \Sigma^{-1} G,
$$

where $\Sigma^{-1}=\operatorname{diag}\left[1 / \sigma_{1}, 1 / \sigma_{2}, \ldots, 1 / \sigma_{n}\right]$. If the determinant of $I$ is non-zero, then $I^{-1}$ is a lower bound to the covariance matrix of any unbiased estimates of $\theta$. We want the covariance matrix to be small. In this paper we use D-optimization, which depends on the maximization of $\operatorname{det}(I)$. To formulate a gradient-based algorithm, we formulate the following problem:

Problem 1. Find derivatives

$$
\frac{\partial \operatorname{det}(I)}{\partial t_{i}}, \quad i=1,2, \ldots, n .
$$

Note that $\operatorname{det}(I)$ is a function of the sensitivity matrix (7), so the problem stated above is to find the "sensitivity function of the function of other sensitivity functions". In the 
next section, the solution to Problem 1 derived by (Fujarewicz, 2007; 2008) will be briefly presented.

\section{Problem solution}

For a particular time moment $t_{i}$, one may write

$$
\frac{\partial \operatorname{det}(I)}{\partial t_{i}}=\sum_{j=1}^{p} \frac{\partial \operatorname{det}(I)}{\partial\left(\partial g_{i}^{0} / \partial \theta_{j}\right)} \cdot \frac{\partial\left(\partial g_{i}^{0} / \partial \theta_{j}\right)}{\partial t_{i}} .
$$

Let us denote the first factor under the sum (10) by $q_{j i}$ and the second factor by $r_{j i}$. Then, let us build matrices $Q=\left[q_{i j}\right], R=\left[r_{i j}\right], \quad Q, R \in \mathbb{R}^{n \times p}$.

It can be shown that the whole matrix $Q$ may be calculated as follows:

$$
Q=2 \Sigma^{-1} G \operatorname{adj}(I)
$$

The element $r_{i j}$ is the derivative with respect to time of the output of the following sensitivity model (model for variations in all variables around the nominal trajectories) for the original model (3), (4):

$$
\begin{aligned}
& \dot{\bar{x}}=f_{x}(t) \bar{x}+f_{\theta}(t) \bar{\theta}, \quad \bar{x}(0)=0, \\
& \bar{y}=g_{x}(t) \bar{x}
\end{aligned}
$$

taken at time $t_{i}$, where the sensitivity is calculated for $\theta_{j}$, which means the variation $\bar{\theta}$ is a vector of zeros except one element number $j$ which equals 1 . Unfortunately, practical usage of (12) requires numerical derivation with respect to time. Instead of this, it is possible to differentiate the second equation in (12) with respect to time, which gives the following formula:

$$
r_{i j}=\dot{x}^{T}\left(t_{i}\right) g_{x x}\left(t_{i}\right) \bar{x}\left(t_{i}\right)+g_{x}\left(t_{i}\right) \dot{\bar{x}}\left(t_{i}\right),
$$

which does not require any numerical derivation because $\dot{x}, \bar{x}$ and $\dot{\bar{x}}$ appear in the original model (3), (4) and in the sensitivity model (12) for $\theta_{j}$.

In order to maximize $\operatorname{det}(I)$, one can combine the results (13) with (11), compute all derivatives $(9)$ and use any gradient-based optimization algorithm.

\section{Sampling optimization for the model of the $\mathrm{NF} \kappa \mathrm{B}$ pathway}

We assumed that only ten parameters of the model (1) were estimated - the same parameters which were fitted in the papers by Lipniacki et al. (2004) and Fujarewicz et al. (2007). The values of parameters estimated by Fujarewicz et al. (2007) were used as nominal ones. The gradient of $\operatorname{det}(I)$ was determined using formulas presented in the previous section. It was used as an input of the conjugated gradient optimization procedure in Matlab.
5.1. Equally distributed starting plan. The initial sampling schedule is presented in Fig. 2 We started with 11 equally distributed times for all five measured variables. For such a schedule, $\operatorname{det}(I)$ was equal to

$$
\operatorname{det}\left(I_{\text {init }}\right)=5.35 \cdot 10^{32} \text {. }
$$

The optimization procedure found an optimal design that is shown in Fig. 3. For this solution, $\operatorname{det}(I)$ increased:

$$
\operatorname{det}\left(I_{\mathrm{opt}}\right)=1.49 \cdot 10^{38} \text {. }
$$

One can see that the number of time points decreased. For all five measured variables these numbers are less than the initial value of 11 . This means that some measurements, according to the optimal plan, should be taken at the same time. Digits shown in Fig. 3 denote numbers of replicates of measurements at the same time moment. This phenomenon is known from the literature for deterministic (Box, 1968) and stochastic (Pronzato and Walter, 1985) cases. In our previous paper (Fujarewicz, 2008), where a simple metabolic pathway (Kutalik et al., 2004) was analyzed, such replicates were also demonstrated. The obtained distinct points are called supporting points. If the so-called continuous design (Kiefer, 1961) is sought, there are known limits on the number $L$ of supporting points:

$$
p \leq L \leq \frac{p(p+1)}{2} .
$$

In continuous design we look for frequencies of measurements at supporting points, rather than their multiplicities. In practice, this requires a very large number of measurements (ideally, an infinite number). Of course, in the case of cell signalling pathways we are looking for a discrete design, because the number of measurements is strongly limited. Nevertheless, we may use the relation (16), which in our case gives

$$
10 \leq L \leq 55
$$

to verify the obtained number of supporting points. In the results presented in Fig. 3, the total number of supporting points is 34 .

Another question should also be posed: Is the $o b$ tained design globally optimal or only locally optimal? To answer this question, let us perform several optimization runs with randomly chosen starting points and check whether all these optimizations give the same result. Figure 4 presents results of ten optimization runs ( 20 points in each run) for the first measured variable: IKKa. There are six distinct time points. The first three points ale very close (cf. Fig. 3).

From Fig. 4 , one can see that

- supporting points are repetitive, 

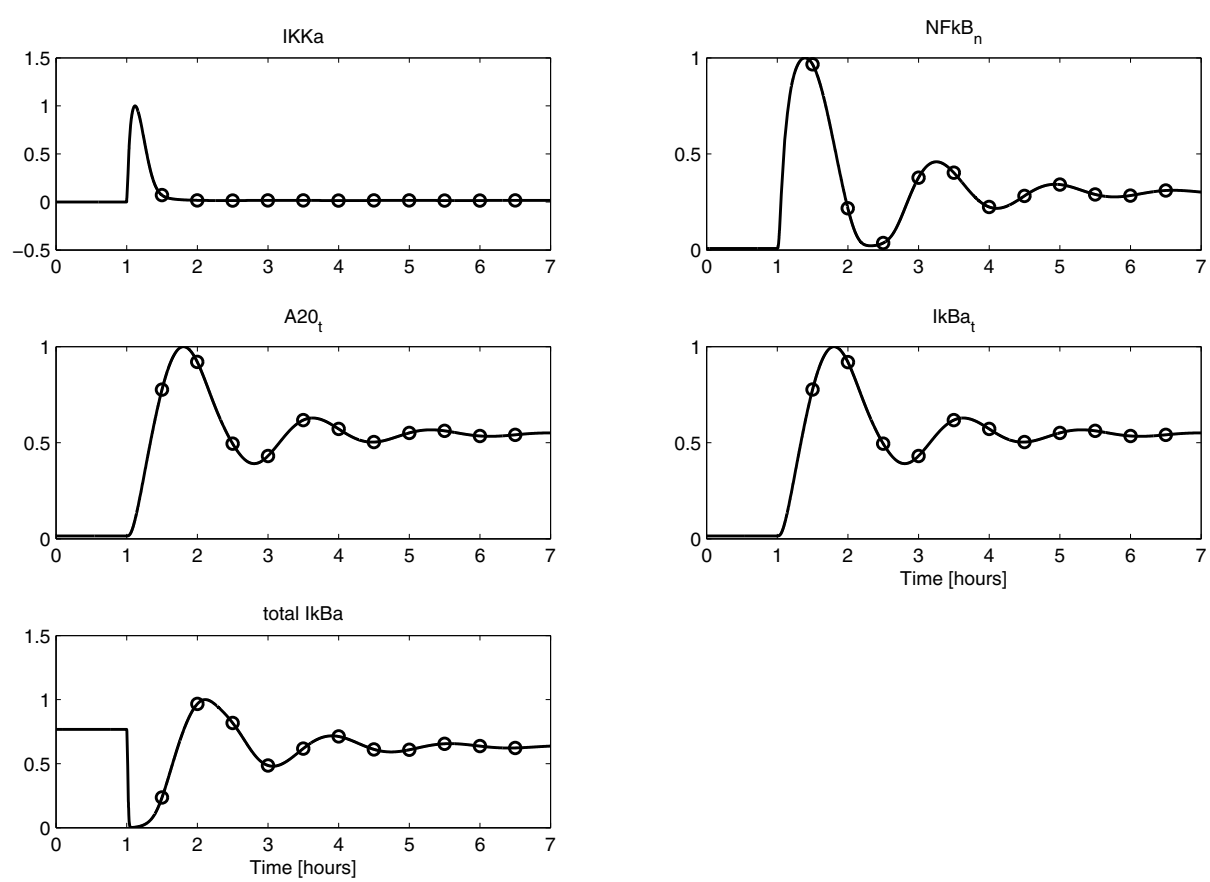

Fig. 2. Initial sampling schedule: 11 equally distributed times for all five measured outputs.
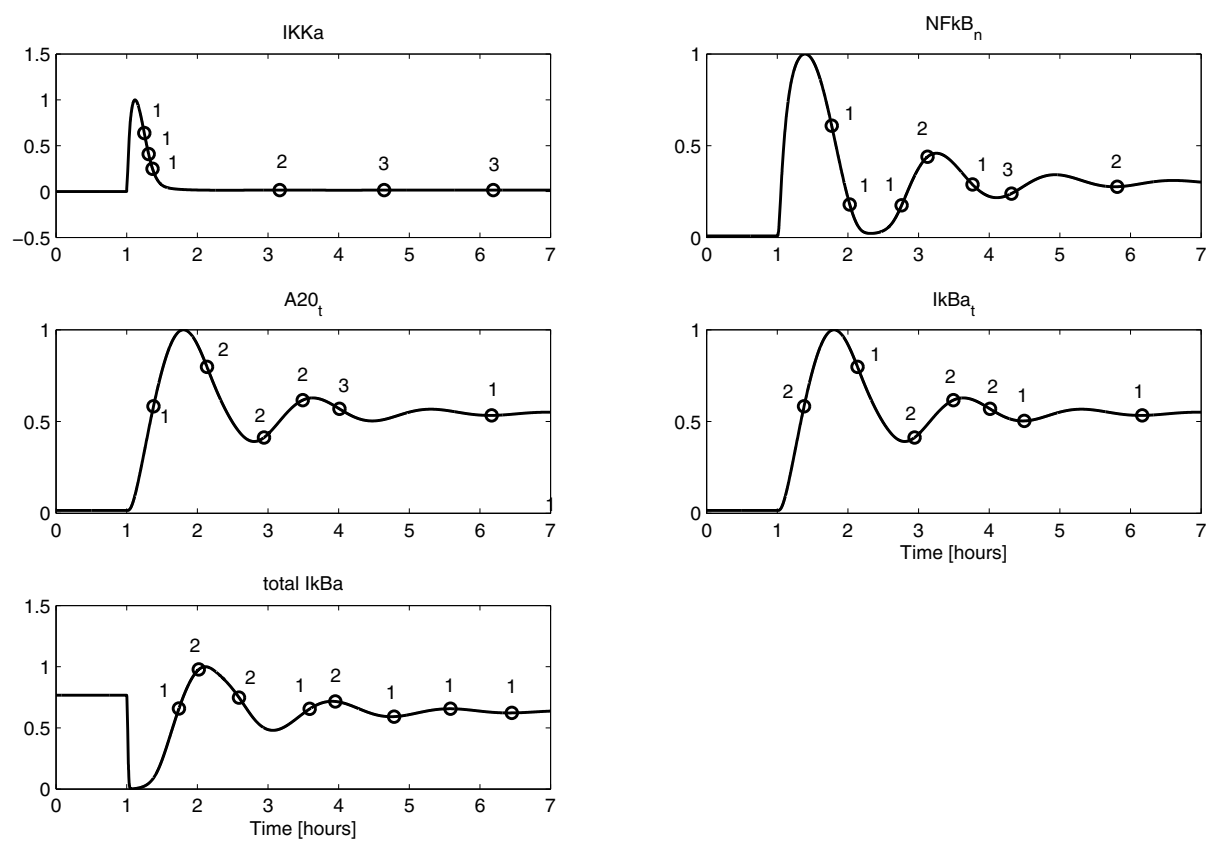

Fig. 3. Optimized sampling schedule. Digits denote the numbers of replicates of measurements at the same time moment. 


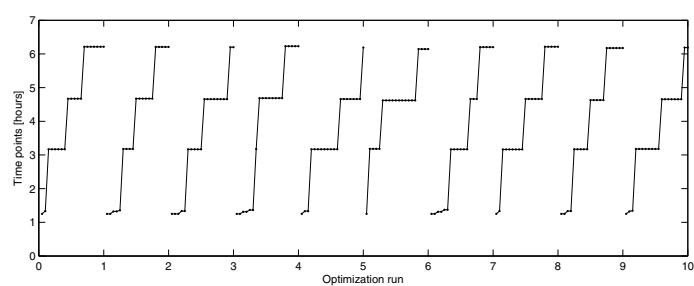

Fig. 4. Optimized plans for ten independent optimization runs starting from random designs.

- multiplicities of supporting points are not repetitive.

In all cases, designs are different and so are the values of $\operatorname{det}(I)$. The gradient-based algorithm used does not produce a unique optimal solution, but it may be used to find all supporting points if the starting design is "dense" enough. All of the above deliberations let us formulate a two-stage optimization algorithm:

1. Use gradient based algorithm with dense starting plan in order to find all distinct supporting points.

2. Use any search algorithm to find multiplicities of supporting points.

5.2. Supporting points detection. In order to find all supporting points, we performed gradient based optimization starting with equally distributed 51 points per one variable (255 in total). The obtained results are collected in Table 1

Table 1. Supporting points (times given in hours) and corresponding multiplicities of the optimal plan for all five measured variables. Note that for some supporting points their multiplicities are equal to zero.

\begin{tabular}{|c|c|c|c|c|}
\hline$I K K a$ & $N F \kappa B$ & $A 20_{t}$ & $I \kappa B a_{t}$ & total $I \kappa B a$ \\
\hline \hline $1.25(5)$ & $1.76(1)$ & $1.38(5)$ & $1.38(2)$ & $1.70(4)$ \\
$1.31(3)$ & $2.01(4)$ & $2.11(0)$ & $2.11(1)$ & $1.86(0)$ \\
$1.36(3)$ & $2.75(2)$ & $2.93(0)$ & $2.93(0)$ & $2.01(3)$ \\
$3.16(0)$ & $3.13(2)$ & $3.48(0)$ & $3.48(4)$ & $2.59(3)$ \\
$4.64(0)$ & $3.78(0)$ & $3.97(4)$ & $3.97(1)$ & $3.58(1)$ \\
$6.18(0)$ & $4.36(2)$ & $4.48(2)$ & $4.48(3)$ & $3.87(0)$ \\
& $5.80(0)$ & $6.17(0)$ & $6.17(0)$ & $4.79(0)$ \\
& & & & $6.47(0)$ \\
\hline
\end{tabular}

It can be seen that there are six, seven, seven, seven and eight distinct supporting points for five measured variables, respectively. The numbers in brackets denote optimal multiplicities for all time points whose optimization is discussed in the next section in what follows.

5.3. Optimization of the supporting point multiplicities. The problem of finding optimal multiplicities for given supporting points is a positive integer programming one. There are many heuristic algorithms to solve such a problem. We used probably the simplest one (Fedorov, 1972), which depends on repetitive exchange of two points and checking whether $\operatorname{det}(I)$ increased. The exchange depends on incrementing (by 1 ) the multiplicity of one supporting point and decrementing the multiplicity of another one for the same variable. We started with random multiplicities and then inspected all possible pairs sequentially until none exchange gave an improvement in the value of $\operatorname{det}(I)$.

Once more the question of the convergence should be addressed. And once more the answer is negative: there is no guarantee that a single-pair exchange leads to a global optimum. To check it out, 300 point multiplicity optimization runs were performed. Figure 5 presents values of maximized $\operatorname{det}(I)$. All of the obtained values belong to the interval $\left[1.8133 \cdot 10^{40}, 1.8671 \cdot 10^{40}\right]$. This interval is relatively narrow. Moreover, in more than $1 / 3$ cases, $\operatorname{det}(I)$ is very close to the upper limit. These sub-optimal values of $\operatorname{det}(I)$ correspond to different designs. The best one is described in Table 1 where multiplicities are given in brackets and presented in Fig. 6. Note that there are a set of designs that are "very close" to the best one in the sense of the value of $\operatorname{det}(I)$.

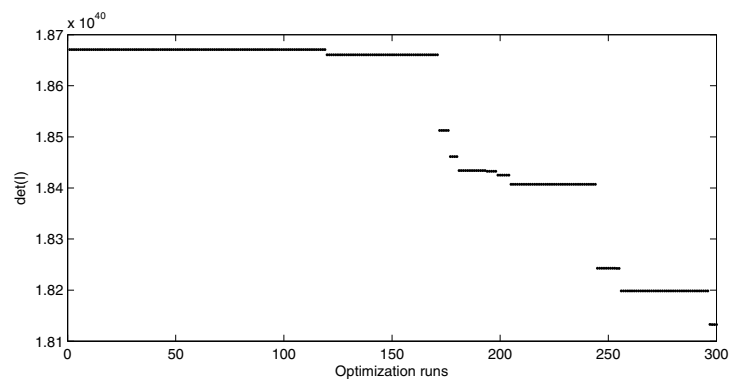

Fig. 5. Sorted values of $\operatorname{det}(I)$ obtained in 300 multiplicity optimization with a random start.

\section{Conclusions}

The obtained result - the best D-optimized design for the $\mathrm{NF} \kappa \mathrm{B}$ signaling pathway-may be non-intuitive, especially for biologists performing experiments. The result is strict from a mathematical point of view. Moreover, for cell populations it is possible to obtain replicates of measurements at the same time moments (one measurementone dish/population).

Nevertheless, intuition tells us that with a decreased number of distinct times of measurements we lose some information about population behavior between the times of measurements. It should be stressed that D-optimum designs (like most other plan optimizations) focuse only on proper parameter estimation. All others information 


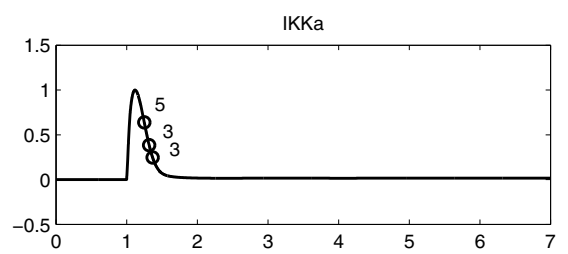

$\mathrm{A} 2 \mathrm{O}_{\mathrm{t}}$

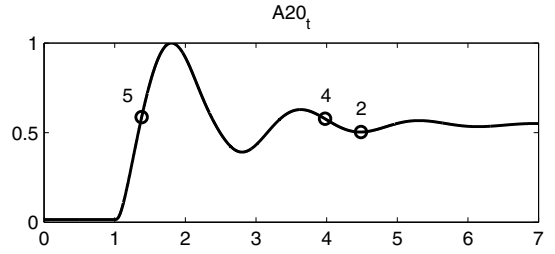

total IkBa

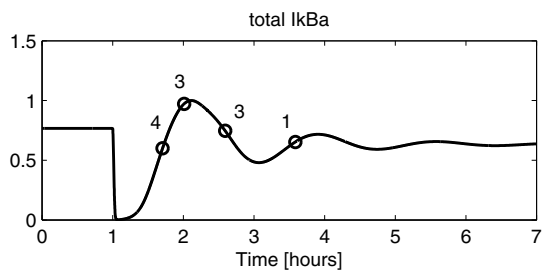

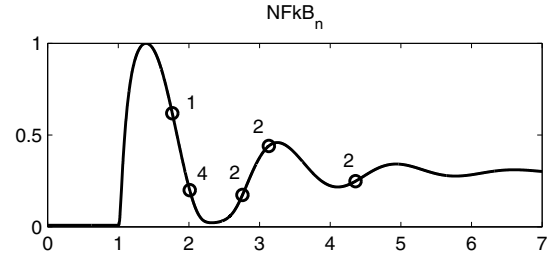

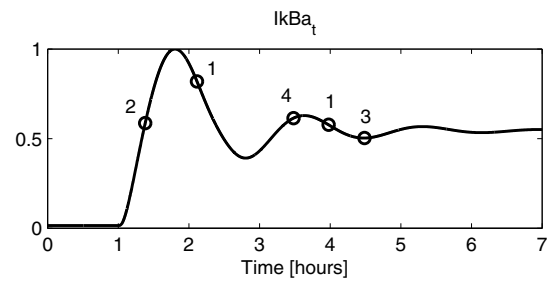

Fig. 6. Final best plan obtained with multiplicity optimization.

is assumed to be known, especially the structure of the model. If one is not sure about the model structure (form of ODEs), then the so-called discriminating experiments should be performed first to select from among all possible hypothetic models.

Another aspect of strong assumptions for Doptimality is also important. There is the assumption about nominal parameters values for which the optimal design is optimized. The aim of experiment design is to discover true parameter values, which means that they are more or less unknown. The nominal values may be parameter estimates obtained from data collected according to the preceding optimized design. Such an iterative approach is a sequential design (Chernoff, 1972).

Most known approaches to experiment design assume some model of uncertainty only for the measured dependent variable. The independent variable (time in the case of dynamical models) is assumed to be known. In the case of cell signaling pathways, taking into account a way in which the experiments are performed, there is some evident (human factor, temperature-dependent speed of reactions, etc.) uncertainty about the time of particular measurements. Modeling uncertainties for both dependent and independent variables may improve the quality of optimized identification designs.

\section{Acknowledgment}

This work has been supported by the Polish Ministry of Science and Higher Education under Grant No. N514
411936. The article is an extended version of a work presented during the 15th National Conference on Application of Mathematics in Biology and Medicine, held in Szczyrk, Poland, in 2009 (Fujarewicz, 2009).

\section{References}

Box, G.E.P. and Lucas, H.L. (1959). Design of experiments in nonlinear situations, Biometrika 46(1/2): 77-90.

Box, M.J. (1968). The occurrence of replications in optimal designs of experiments to estimate parameters in non-linear models, Journal of the Royal Statistical Society. Series B 30(2): 290-302.

Chernoff, H. (1972). Sequential Analysis and Optimal Design, SIAM, Philadelphia, PA.

D'Argenio, D.Z. (1981). Optimal sampling times for pharmacokinetic experiments, Journal of Pharmacokinetics and Biopharmaceutics 9(6): 739-756.

de Jong, H. (2002). Modeling and simulation of genetic regulatory systems: A literature review, Journal of Computational Biology 9(1): 67-103.

DiStefano, J.J. (1981). Optimized blood sampling protocols and sequential design of kinetic experiments, American Journal of Physiology 9(240): R259-R265.

Fedorov, V.V. (1972). Theory of Optimal Experiments, Academic Press, New York, NY.

Fujarewicz, K. (2007). Planning identification experiments for cell signaling pathways using sensitivity analysis, Proceedings of the 23rd IFIP Conference on System Modelling and Optimization, Cracow, Poland, pp. 262-263. 
Fujarewicz, K. (2008). Optimal scheduling for parameter estimation of cell signaling pathway models-A gradient approach, Proceedings of the 25th IASTED International Multi-Conference on Biomedical Engineering, Innsbruck, Austria, pp. 232-236.

Fujarewicz, K. (2009). Planning identification experiments for nfkb signaling pathway, Proceedings of the 15th National Conference on Application of Mathematics in Biology and Medicine, Szczyrk, Poland, pp. 64-53.

Fujarewicz, K. and Galuszka, A. (2004). Generalized backpropagation through time for continuous time neural networks and discrete time measurements, in L. Rutkowski, J. Siekmann, R. Tadeusiewicz and L. A. Zadeh (Eds.) Artificial Intelligence and Soft Computing-ICAISC 2004, Lecture Notes in Computer Science, Vol. 3070, Springer-Verlag, Berlin, pp. 190-196.

Fujarewicz, K., Kimmel, M., Lipniacki, T. and Swierniak, A. (2007). Adjoint systems for models of cell signalling pathways and their application to parameter fitting, IEEE/ACM Transactions on Computational Biology and Bioinformatics 4(3): 322-335.

Goodwin, G.C. and Payne, R.L. (1977). Dynamic System Identification: Experiment Design and Data Analysis, Academic Press, New York, NY.

Hoffman, A., Levchenko, A., Scott, M.L. and Baltimore, D. (2002). The i $\kappa b-n f-\kappa b$ signaling module: Temporal control and selective gene activation, Science 298: 1241-1245.

Jacquez, J. (1998). Designs of experiments, Journal of Franklin Institute 335(2).

Jacquez, J. and Greif, P. (1985). Numerical parameter identifiability and estimability: Integrating identifiability, estimability, and optimal sampling design, Mathematical Biosciences 77: 201-227.

Kiefer, J. (1961). Optimum designs in regression problems, II. The Annals of Mathematical Statistics 32(1): 298-325.
Kutalik, Z., Cho, K. and Wolkenhauer, O. (2004). Optimal sampling time selection for parameter estimation in dynamic pathway modeling, BioSystems 75(1-3): 43-55.

Lee, E., Boone, D., Chai, S., Libby, S., Chien, M., Lodolce, J. and Ma, A. (2000). Failure to regulate tnf-induced nf$\kappa \mathrm{b}$ and cell death responses in a20-deficient mice, Science 289(5488): 2350-2354.

Lipniacki, T., Paszek, P., Brasier, A.R., Luxon, B. and Kimmel, M. (2004). Mathematical model of $n f-\kappa b$ regulatory module, Journal of Theoretical Biology 228(2): 195-215.

Pronzato, L. and Walter, E. (1985). Robust experiment design via stochastic approximation, Mathematical Biosciences 75: $103-120$.

Tod, M. and Rocchisani, J.M. (1997). Comparison of ed, eid and api criteria for the robust optimization of sampling times in pharmacokinetics, Journal of Pharmacokinetics and Biopharmaceutics 25(4): 515-537.

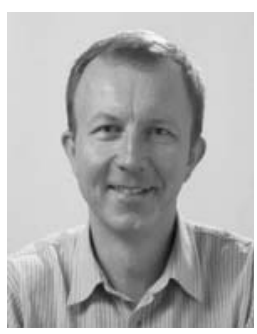

Krzysztof Fujarewicz was born in Poland in 1967. He received the M.Sc. degree in automatic control from the Silesian University of Technology in Gliwice in 1992, and the Ph.D degree in automatic control and robotics from the same university in 1999. In 1999, he became an assistant professor at the Institute of Automatic Control, Silesian University of Technology, Gliwice. In the years 2003-2006, he was several times a visiting researcher at Rice University, Houston, USA. His main research interest is in various applications of neural networks, identification and optimal control of non-linear systems, classification, clustering and feature selection for biomedical data.

Received: 18 January 2010 Revised: 9 June 2010 